\title{
İndirgenmiş Diferansiyel Dönüşüm Metodu ile Fokker-Planck Denkleminin Yaklaşık Çözümü
}

\author{
Derya ARSLAN
}

Bitlis Eren Üniversitesi, Fen Edebiyat Fakültesi, Matematik Bölümü 13200, Bitlis, Türkiye

Geliş / Received: 05/08/2018, Kabul / Accepted: 19/11/2018

\begin{abstract}
$\ddot{O} \mathbf{z}$
Bu çalışmada, kısmi türevli denklemler sınıfına ait Fokker-Planck denkleminin etkili bir nümerik yöntem olan indirgenmiş diferansiyel dönüşüm metodu ile yaklaşık analitik çözümü incelenir. Bu çözüm seri formda oluşur. Tam ve yaklaşık çözümde $x$ ve $t$ 'ye farklı değerler verilerek grafikler çizilir, elde edilen sayısal sonuçlar tablolar üzerinde gösterilir. Tüm bu veriler, indirgenmiş diferansiyel dönüşüm metodunun, sayısal terimleri hızlı ve kolay bir şekilde hesapladığını, üstelik yakınsak analitik bir yaklaşım sağladığını gösterir.
\end{abstract}

Anahtar Kelimeler: İndirgenmiş diferansiyel dönüşüm metodu, Fokker-Planck denklemi, Seri çözüm, Yaklaşık çözüm.

\section{The Approximate Solution of Fokker-Planck Equation with Reduced Differential Transform Method}

\begin{abstract}
Abctract
In this study, the approximate analytical solution of the Fokker-Planck equation for the class of partially differential equations is examined by using reduced differential transformation method which is an effective numerical method. This solution occurs in the form of a series. The figures are drawn for different values of $x$ and $t$ in the exact and approximate solution, the numerical results are shown on the tables. All these data show that the reduced differential transform method computes quickly and easily numerical terms, and also provides a convergent analytical approach.
\end{abstract}

Keywords: Reduced differential transform method, Fokker-Planck equation, series solution, approximate solution.

\section{Giriş}

Son y1llarda matematiksel fizikteki önemi nedeniyle Fokker-Planck denklemine birçok fizikçi ve matematikçi bilim insanı dikkat çekmiştir (Bhrawy vd., 2014; Dreger vd., 2005; Hesam vd., 2012; Narayanan ve Kumar, 2006; Kazem vd., 2012; Kım ve Tranquilli, 2008; Koide, 2017; Lakestanı ve Dehghan, 2009; Sadighi vd., 2007; Tatari vd., 2007). Fokker-Planck denklemi lojistik nüfus artışı, alev yayılımı, nörofizyoloji, otokatalitik kimyasal reaksiyon, nükleer reaktör teori, katı hal fiziği, kuantum optiği, kimyasal fizik, teorik biyoloji ve devre teorisi alanlarını içeren doğal bilim uygulamalarına sahiptir. Fokker-Planck denklemini çözmek için analitik ve nümerik olarak birçok yöntem kullanılmıştır. Bunlardan bazıları:
Tatari vd. (2007) Adomian ayrıştırma metodu, Hesam vd. (2012) iki boyutlu diferansiyel dönüşüm metodu, Bhrawy vd. (2014) kollokasyon metodu, Sadighi vd. (2007) homotopi pertürbasyon metodu, Biazar vd. (2010) varyasyonel iterasyon metodu, Dreger vd. (2005) varyasyonel pertürbasyon metodu ile Narayanan ve Kumar (2006) sonlu elemanlar ve sonlu fark metodu çalışmalarıdır. Çalışmanın amacında, bu literatür bilgileri göz önünde bulundurularak Fokker-Planck denklemi için kullanılmış bu yöntemler dişında farklı bir metot olarak indirgenmiş diferansiyel dönüşüm metodu tercih edilmiştir. İndirgenmiş diferansiyel dönüşüm metodu da birçok lineer ve lineer olmayan problemlerin çözümünde kullanılmıştır (Benhammouda 
vd., 2014; Cakır ve Arslan, 2015; Cakır ve Arslan, 2016; Gupta, 2011; Haghbin ve Hesam, 2012; Hosseinzadeh ve Salehpour, 2013; Keskin ve Oturanç, 2010; Mohamed ve Gepreel, 2017; Rawashdeh, 2013; Saravanan ve Magesh, 2013; Srivastava vd., 2014; Yıldırım vd., 2012). Öneri olarak, referanslarda verilen diğer farklı metotlarla çözülmüş Pseudoparabolic ve üçüncü mertebe kısmi türevli diferansiyel denklemler için de güvenle tercih edilebilir (Amiraliyev vd., 2017; Amirali vd., 2014, Kudu ve Amiariyeva, 2014). Metodun işleyiş şeması, $t$ - zaman değişkenine göre Fokker-Planck denkleminin her bir terimine karşılık gelen diferansiyel dönüşümler, Fokker-Planck denkleminde yerine yazılarak tekrar bağıntısı elde edilir. $\mathrm{Bu}$ tekrar bağıntısından elde edilen $Y(1), \ldots, Y(k)$ diferansiyel dönüşüm katsayıları $y(x, t)$ ters diferansiyel dönüşümünde yazılır ve seri çözümüne ulaşılır. En son aşamada ise bu seri çözüm ile tam çözüm karşılaştırılarak metodun etkinliği ve uygulanabilirliği ispatlanmış olur. $\mathrm{Bu}$ çalışma şöyle organize edilir: Sırasıyla, Fokker-Planck denkleminin özellikleri verilir. İndirgenmiş diferansiyel dönüşüm metodunun tanımı yapılır. Son olarak da indirgenmiş diferansiyel dönüşüm metodu sadece 4 iterasyon ile örnek problemlere uygulanır. Bu uygulama sonucu bulunan seri çözümünde, $x$ ile $t$ 'ye verilen farklı değerler için tam ve yaklaşık çözüm değerleri grafikler ve tablolarla sunulur.

\subsection{Fokker-Planck denklemi ve indirgenmiş diferansiyel dönüşüm metodu}

Fokker-Planck denklemi, ilk olarak Fokker ve Planck tarafından parçacıkların Brownian hareketini tanımlamak için kullanıldı (Risken, 1989). Fokker-Planck denkleminin forward Kolmogorov olarak da bilinen genel formu,

$$
\begin{aligned}
& \frac{\partial u}{\partial t}=\left[-\frac{\partial}{\partial x} A(x)+\frac{\partial^{2}}{\partial x^{2}} B(x)\right] u, \\
& u(x, 0)=f(x), \quad x \in R,
\end{aligned}
$$

burada $B(x)>0$ difüzyon katsayısı, $A(x)$ ise drift katsayısı olarak tanımlanır. Bu katsayıların durumuna göre Fokker-planck denkleminde drift ve difüzyon katsayıları:

a) Sadece $x$ - konum değişkenine bağlı olabilir.

b) $A(x, t)=\alpha$ ve $B(x, t)=\beta$ sabitleri olabilir.

c) $t$ - zaman değişkenine bağlı olabilir.

$$
\frac{\partial u}{\partial t}=\left[-\frac{\partial}{\partial x} A(x, t)+\frac{\partial^{2}}{\partial x^{2}} B(x, t)\right] u .
$$

d) Risken (1989) tarafından verilen backward Kolmogorov denklemi olarak bilinen aşağıdaki formda olabilir.

$$
\frac{\partial u}{\partial t}=\left[-A(x, t) \frac{\partial}{\partial x}+B(x, t) \frac{\partial^{2}}{\partial x^{2}}\right] u .
$$

e) Özel formu olan; drift katsayısı lineer, difüzyon katsayısı da sabit olabilir.

Fokker-Planck denkleminin diğer formları çalışmanın kaynaklarında görülebilir. Biz bu çalışmada ilk dört formu indirgenmiş diferansiyel dönüşüm metodu ile inceledik.

İki boyutlu diferansiyel dönüşüm metodu, iki boyutlu kısmi türevli diferansiyel denklemlere $t$ - zaman ve $x$ - konum değişkenine göre ilk kez 1999 yılında Chen ve Ho tarafından uygulanmıştır (Chen ve Ho, 1999). Buna karşılık sadece $t$-zaman değişkenine göre 
uygulanan indirgenmiş diferansiyel dönüşüm metodu, lineer ve lineer olmayan kısmi türevli diferansiyel denklemlerin çözümünde az sayıda iterasyon uygulayarak kısa zamanda çözüme ulaşılması bakımından tercih edilmektedir. Diferansiyel dönüşüm metodunun tanım ve özelliklerinden yararlanarak, indirgenmiş diferansiyel dönüşüm metodunun formunu yazabiliriz (Zhou, 1986; Ayaz, 2003; Chen ve Ho, 1999)

İki bileşenli $u(x, t)$ fonksiyonuna karşılık gelen diferansiyel dönüşüm fonksiyonu $U(h, k)$ olmak üzere, $t-$ zaman değişkenine göre $u(x, t)$ çözümü (Keskin ve Oturanç, 2010)

$$
u(x, t)=\sum_{k=0}^{\infty} U_{k}(x) t^{k}=U_{0}(x)+U_{1}(x) t+U_{2}(x) t^{2}+U_{3}(x) t^{3}+\cdots,
$$

olarak tanımlanır. $u(x, t)^{\prime}$ 'nin $t$ - zaman değişkenine göre indirgenmiş diferansiyel dönüşümü (Keskin ve Oturanç, 2010)

$$
U_{k}(x)=\frac{1}{k !}\left[\frac{\partial^{k}}{\partial t^{k}} u(x, t)\right]_{t=0}
$$

olarak tanımlanır.

$U_{k}(x)$ 'in $t$ 'ye göre indirgenmiş diferansiyel dönüşüm fonksiyonunun tersi (Keskin ve Oturanç, 2010)

$$
u(x, t)=\sum_{k=0}^{\infty} U_{k}(x) t^{k}
$$

şeklinde tanımlanır. (2) ve (3) eşitlikleri kullanılarak aşağıdaki (4) eşitliği elde edilir (Keskin ve Oturanç, 2010):

$$
U_{k}(x)=\sum_{k=0}^{\infty} \frac{1}{k !}\left[\frac{\partial^{k}}{\partial t^{k}} u(x, t)\right]_{t=0} t^{k}
$$

Yukarıda verilen eşitlikler ve birtakım matematiksel işlemlerle indirgenmiş diferansiyel dönüşüm metodunun bazı özellikleri Tablo 1'de verilir (Keskin ve Oturanç, 2010). Bu özellikler Fokker-Planck denkleminin çözümünde kullanılacaktır.

Tablo $1 t$ - zaman değişkenine göre indirgenmiş diferansiyel dönüşümün bazı özellikleri

\begin{tabular}{|l|l|}
\hline \multicolumn{1}{|c|}{ Fonksiyon } & \multicolumn{1}{c|}{ Dönüşüm Karşıı̆ı̆̆ } \\
\hline$u(x, t)$ & $U_{k}(x)=\frac{1}{k !}\left[\frac{\partial^{k}}{\partial t^{k}} u(x, t)\right]_{t=0}$ \\
\hline$w(x, t)=e^{t}$ & $W_{k}(x)=\frac{1}{k !}$ \\
\hline$w(x, t)=\alpha u(x, t), \alpha$ sabittir. & $W_{k}(x)=\alpha U_{k}(x)$ \\
\hline$w(x, t)=\frac{\partial u(x, t)}{\partial t}$ & $W_{k}(x)=(k+1) U_{k+1}(x)$ \\
\hline$w(x, t)=\frac{\partial u(x, t)}{\partial x}$ & $W_{k}(x)=\frac{\partial U_{k}(x)}{\partial x}$ \\
\hline$w(x, t)=\frac{\partial^{2} u(x, t)}{\partial x^{2}}$ & $W_{k}(x)=\frac{\partial^{2} U_{k}(x)}{\partial x^{2}}$ \\
\hline
\end{tabular}




\section{2. İndirgenmiş diferansiyel dönüşüm metodunun Fokker-Planck denklemine uygulanması}

\section{Örnek 1}

$$
\begin{aligned}
& \frac{\partial u}{\partial t}=\frac{\partial u}{\partial x}+\frac{\partial^{2} u}{\partial x^{2}}, \\
& u(x, 0)=x, \quad x \in R
\end{aligned}
$$

Fokker-Planck denkleminde drift ve difüzyon katsayıları sabit biçimde $A(x)=-1, B(x)=1$ olarak verilmiştir. Ayrıca $f(x)=x$ olup (5)-(6) denkleminin tam çözümü ise

$$
u(x, t)=x+t,
$$

biçimindedir (Biazar vd., 2010). İndirgenmiş diferansiyel dönüşüm metodu kullanarak (5)-(6) Fokker-Planck denkleminde $t$ - zaman değişkenine göre her bir terimine ve $u(x, 0)=x$ başlangıç durumuna karşılık gelen diferansiyel dönüşümler

$$
\begin{aligned}
& \frac{\partial u}{\partial t} \rightarrow U_{k}(x)=(k+1) U_{k+1}(x), \\
& \frac{\partial u}{\partial x} \rightarrow \frac{\partial U_{k}(x)}{\partial x} \\
& \frac{\partial^{2} u}{\partial x^{2}} \rightarrow \frac{\partial^{2} U_{k}(x)}{\partial x^{2}} \\
& x \rightarrow U_{0}(x)=x
\end{aligned}
$$

bulunur. $\mathrm{Bu}$ diferansiyel dönüşümler (5) denkleminde yerlerine yazılırsa aşağıdaki tekrar bağıntısı elde edilir:

$$
U_{k+1}(x)=\frac{1}{k+1}\left[\frac{\partial U_{k}(x)}{\partial x}+\frac{\partial^{2} U_{k}(x)}{\partial x^{2}}\right] .
$$

Burada $k=0,1,2,3, \ldots$ değerleri için 4 iterasyon ile aşağıdaki diferansiyel dönüşüm katsayıları bulunur:

$$
U_{0}(x)=x, \quad U_{1}(x)=1, \quad U_{2}(x)=0, \quad U_{3}(x)=0, \quad U_{4}(x)=0,
$$

bu diferansiyel dönüşüm katsayıları (1) denkleminde yerlerine yazılırsa seri çözümü bulunur.

$$
u(x, t)=\sum_{k=0}^{\infty} U_{k}(x) t^{k}=x+t+0 t^{2}+0 t^{3}+0 t^{4}=x+t,
$$

Tablo $2 t=0.01$ değeri için tam çözüm, yaklaşık çözüm ve hata değerleri

\begin{tabular}{|c|c|c|c|}
\hline $\mathrm{X}$ & Tam çözüm & Yaklaşı çözüm & Hata \\
\hline-1.0 & -0.99 & -0.99 & 0 \\
\hline-0.6 & -0.59 & -0.59 & 0 \\
\hline-0.2 & -0.19 & -0.19 & 0 \\
\hline 0 & 0.01 & 0.01 & 0 \\
\hline 0.2 & 0.21 & 0.21 & 0 \\
\hline 0.6 & 0.61 & 0.61 & 0 \\
\hline
\end{tabular}




\begin{tabular}{|l|l|l|l|}
\hline 1.0 & 1.01 & 1.01 & 0 \\
\hline
\end{tabular}

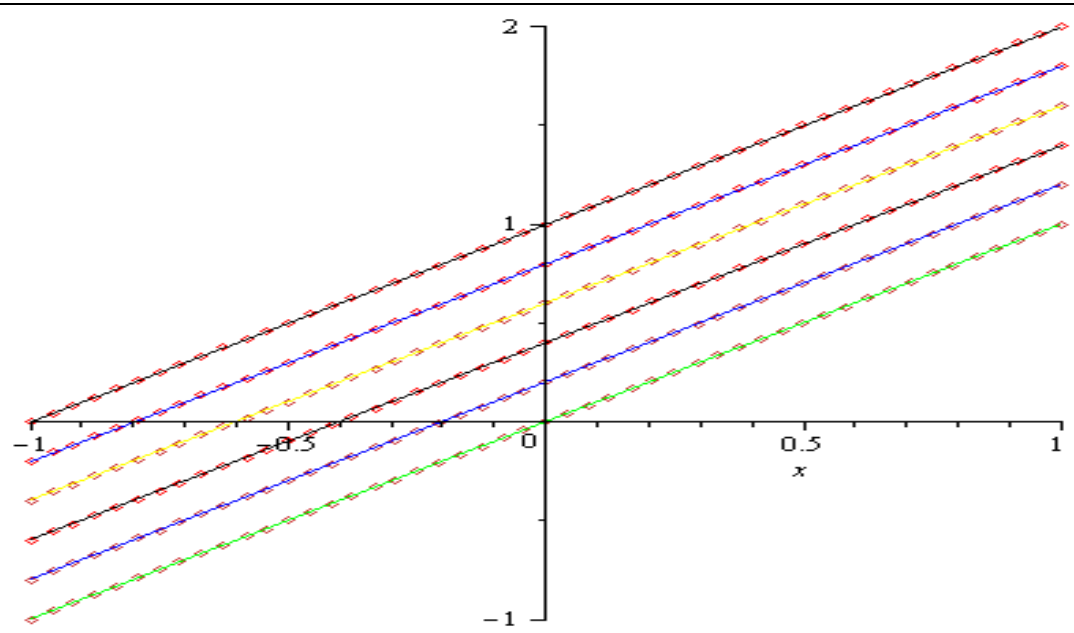

Şekil $1 t=0,0.2,0.4,0.6,0.8,1$ için tam ve yaklaşık çözüm doğrularının karşılaştırması

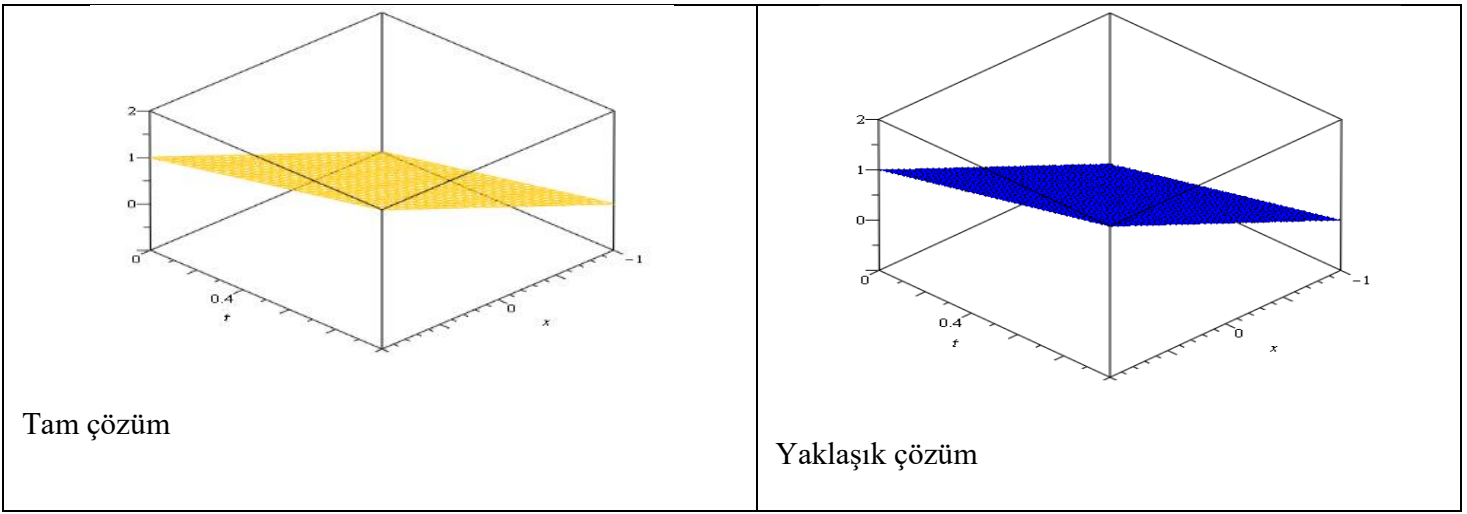

Şekil $2 x$ ve $t$ değerleri için tam ve yaklaşık çözüm eğrileri

Tablo 1'de, $t=0.01$ ve $x$ 'in farklı değerleri için tam çözüm, yaklaşık çözüm ve hata değerleri gösterilmiştir. (5)-(6) Fokker-Planck denkleminin tam çözümünden ve indirgenmiş diferansiyel dönüşüm metodundan elde edilen yaklaşık çözüm eğrileri Şekil 1'de $\operatorname{artan} t-$ değerlerine göre (doğrular, en alttan en üste doğru dizilir) karşılaştırılır. $x$ ve $t$ değerleri için tam ve yaklaşık çözüm eğrileri de Şekil 2 ile gösterilir.

\section{Örnek 2}

$$
\begin{gathered}
\frac{\partial u}{\partial t}=-\frac{\partial u}{\partial x}\left(-\tanh x+e^{t} \sinh x\right)+\frac{\partial^{2} u}{\partial x^{2}}\left(e^{t} \cosh x\right), \\
u(x, 0)=\sinh x, \quad x \in R,
\end{gathered}
$$

formundaki Fokker-Planck denkleminin tam çözümü

$$
u(x, t)=e^{t} \sinh x .
$$

(7)-(8) denkleminde $f(x)=\sinh x, \quad A(x, t)=-\tanh x+e^{t} \sinh x$ drift ve $B(x, t)=$ $e^{t} \cosh x$ difüzyon katsayılarıdır (Hesam vd., 2012). Değişken katsayılı bu denklemin her bir terimine ve başlangıç durumuna karşılık gelen diferansiyel dönüşümler

$$
\begin{aligned}
\frac{\partial u}{\partial t} \rightarrow U_{k}(x) & =(k+1) U_{k+1}(x), \\
\frac{\partial u}{\partial x} & \rightarrow \frac{\partial U_{k}(x)}{\partial x}
\end{aligned}
$$




$$
\begin{gathered}
\frac{\partial^{2} u}{\partial x^{2}} \rightarrow \frac{\partial^{2} U_{k}(x)}{\partial x^{2}}, \\
\sinh x \rightarrow U_{0}(x)=\frac{\sinh x}{k !},
\end{gathered}
$$

olarak bulunur. Bu diferansiyel dönüşümler (7) denkleminde yerlerine yazılırsa

$$
U_{k+1}(x)=\frac{1}{k+1}\left[\left(\tanh x-\frac{\sinh x}{k !}\right) \frac{\partial U_{k}(x)}{\partial x}+\left(\frac{\cosh x}{k !}\right) \frac{\partial^{2} U_{k}(x)}{\partial x^{2}}\right]
$$

tekrar bağıntısı elde edilir. Burada $k=0,1,2,3, \ldots$ değerleri için

$U_{0}(x)=\sinh x, \quad U_{1}(x)=\sinh x, \quad U_{2}(x)=\frac{\sinh x}{2 !}, \quad U_{3}(x)=\frac{\sinh x}{3 !}, \quad U_{4}(x)=\frac{\sinh x}{4 !}$,

diferansiyel dönüşüm katsayıları 4 iterasyon yapılarak bulunur ve (1) denkleminde yazılırsa

$$
\begin{aligned}
& u(x, t)=\sum_{k=0}^{\infty} U_{k}(x) t^{k}=\sinh x+t \sinh x+t^{2} \frac{\sinh x}{2 !}+t^{3} \frac{\sinh x}{3 !}+\cdots, \\
& u(x, t)=\sinh x\left(1+t+\frac{t^{2}}{2 !}+\frac{t^{3}}{3 !}\right)
\end{aligned}
$$

çözümü elde edilir.

İndirgenmiş diferansiyel dönüşüm metodunun etkinliğini görmek için Tablo 3'te $t=$ 0.01 ve farklı $x$ değerlerine göre tam çözüm, yaklaşık çözüm ve hatanın sayısal sonuçları verilir. Şekil $3, t=0,0.2,0.4,0.6,0.8,1$ farklı zamanları için Fokker-Planck denkleminin farklı noktalarda tam ve yaklaşı çözüm eğrilerini gösterir (yani artan $t$ - değerlerine göre eğriler en alttan en üste doğru sıralanmıştır). Şekil 4 ise farklı zaman ve konumlarda tam çözüm ve yaklaşık çözüm eğrilerini verir.

Tablo $3 t=0.01$ değeri için tam çözüm, yaklaşık çözüm ve hata değerleri

\begin{tabular}{|c|c|c|c|}
\hline $\mathrm{x}$ & Tam çözüm & Yaklaşı çözüm & Hata \\
\hline-1.0 & -1.1870121620 & -1.1870119670 & $0.195 \mathrm{E}-3$ \\
\hline-0.8 & -0.8970315956 & -0.8970314474 & $0.1482 \mathrm{E}-2$ \\
\hline-0.6 & -0.6430520569 & -0.6430619505 & $0.1064 \mathrm{E}-2$ \\
\hline-0.4 & -0.4148804553 & -0.4148803866 & $0.687 \mathrm{E}-4$ \\
\hline-0.2 & -0.2033594629 & -0.2033594293 & $0.336 \mathrm{E}-4$ \\
\hline 0.2 & 0.2033594629 & 0.2033594293 & $0.336 \mathrm{E}-4$ \\
\hline 0 & 0 & 0 & 0 \\
\hline 0.4 & 0.4148804553 & 0.4148803866 & $0.687 \mathrm{E}-4$ \\
\hline 0.6 & 0.6430520569 & 0.6430519505 & $0.1064 \mathrm{E}-2$ \\
\hline 0.8 & 0.8970315956 & 0.8970314474 & $0.1482 \mathrm{E}-2$ \\
\hline 1.0 & 1.1870121620 & 1.1870119670 & $0.195 \mathrm{E}-3$ \\
\hline
\end{tabular}




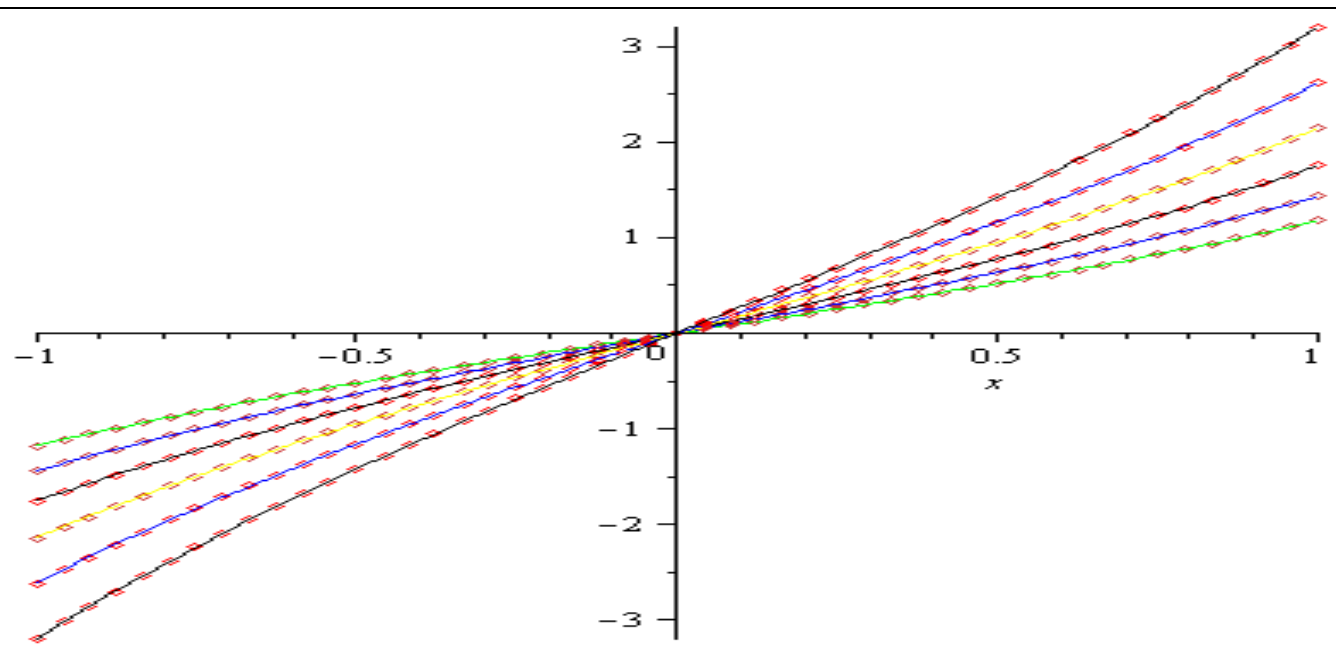

Şekil $3 t=0,0.2,0.4,0.6,0.8,1$ için tam çözüm ve yaklaşı çözüm eğrilerinin karşılaştırması

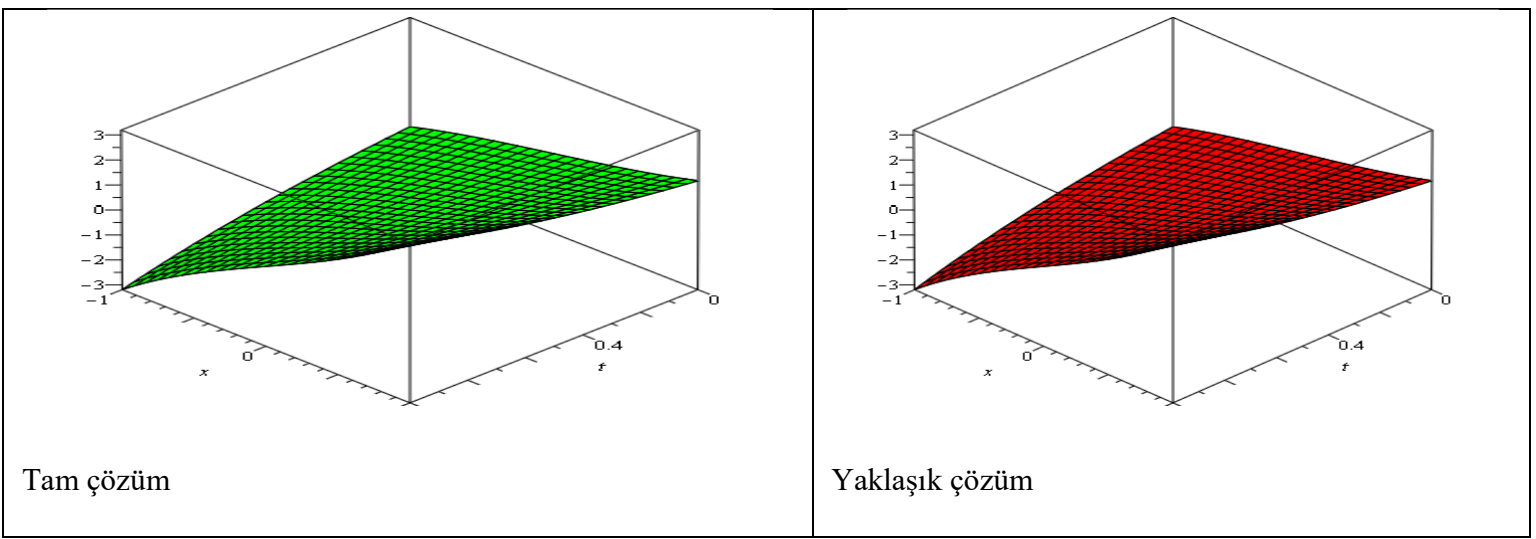

Şekil $4 x$ ve $t$ değerleri için tam ve yaklaş1k çözüm eğrileri

\section{Örnek 3}

$$
\begin{gathered}
\frac{\partial u}{\partial t}=(x+1) \frac{\partial u}{\partial x}+\left(e^{t} x^{2}\right) \frac{\partial^{2} u}{\partial x^{2}}, \\
u(x, 0)=x+1, \quad x \in R,
\end{gathered}
$$

(9)-(10) Fokker-Planck denkleminde değişken katsayılı drift ve difüzyon katsayıları $A(x, t)=$ $-(x+1), B(x, t)=e^{t} x^{2} ; f(x)=x+1$ ve denklemin tam çözümü

$$
u(x, t)=(x+1) e^{t},
$$

formundadır (Hesam vd., 2012). (9)-(10) Fokker-Planck denkleminde $t$ - zaman değişkenine göre her bir terime ve $u(x, 0)=x+1$ başlangıç değerine karşılık gelen diferansiyel dönüşümler aşağıdaki gibidir:

$$
\begin{gathered}
\frac{\partial u}{\partial t} \rightarrow U_{k}(x)=(k+1) U_{k+1}(x), \\
\frac{\partial u}{\partial x} \rightarrow \frac{\partial U_{k}(x)}{\partial x}, \\
\frac{\partial^{2} u}{\partial x^{2}} \rightarrow \frac{\partial^{2} U_{k}(x)}{\partial x^{2}} \\
x+1 \rightarrow U_{0}(x)=x+1 .
\end{gathered}
$$


$\mathrm{Bu}$ diferansiyel dönüşümler (9) denkleminde yerlerine yazılırsa aşağıdaki tekrar bağıntısı bulunur:

$$
U_{k+1}(x)=\frac{1}{k+1}\left[(x+1) \frac{\partial U_{k}(x)}{\partial x}+\left(\frac{x^{2}}{k !}\right) \frac{\partial^{2} U_{k}(x)}{\partial x^{2}}\right]
$$

Burada $k=0,1,2,3, \ldots$ değerleri için diferansiyel dönüşüm katsayıları

$$
U_{0}(x)=x+1, \quad U_{1}(x)=x+1, \quad U_{2}(x)=\frac{x+1}{2 !}, \quad U_{3}(x)=\frac{x+1}{3 !},
$$

biçiminde 4 iterasyon yapılarak elde edilir. $\mathrm{Bu}$ diferansiyel dönüşüm katsayıları (1) denkleminde yerlerine yazılırsa

$$
\begin{aligned}
& u(x, t)=\sum_{k=0}^{\infty} U_{k}(x) t^{k}=x+1+(x+1) t+\frac{(x+1)}{2 !} t^{2}+\frac{(x+1)}{3 !} t^{3}+\cdots \\
& u(x, t)=(x+1)\left(1+t+\frac{t^{2}}{2 !}+\frac{t^{3}}{3 !}\right)
\end{aligned}
$$

çözümü elde edilir.

Tablo 4'te, $t=0.01$ değeri ve farklı $x$ 'ler için (9)-(10) Fokker-Planck denkleminin tam çözüm ve indirgenmiş diferansiyel dönüşüm metodundan elde edilen yaklaşık çözüm verileri ile hata değerleri listelenmiştir. Şekil 5'te $t=0,0.2,0.4,0.6,0.8,1 \quad$ değerlerine göre tam çözüm ve yaklaşık çözüm eğrileri (aşağıdan yukarı doğru sıralanarak) karşılaştırma amacıyla çizilir. Ayrıca, $x$ ve $t$ değerleri için tam ve yaklaşık çözüm eğrileri Şekil 6 ile verilir.

Tablo $4 t=0.01$ değeri için tam çözüm, yaklaşık çözüm ve hata değerleri

\begin{tabular}{|c|c|c|c|}
\hline $\mathrm{x}$ & Tam çözüm & Yaklaşık çözüm & Hata \\
\hline-1.0 & -1.0100501670 & -1.0100501670 & 0 \\
\hline-0.8 & -0.8080401336 & -0.8080401336 & 0 \\
\hline-0.6 & -0.6060301002 & -0.6060301002 & 0 \\
\hline-0.4 & -0.4040200668 & -0.4040200668 & 0 \\
\hline-0.2 & -0.2020100334 & -0.2020100334 & 0 \\
\hline 0 & & & 0 \\
\hline 0.2 & 0.2020100334 & 0.2020100334 & 0 \\
\hline 0.4 & 0.4040200668 & 0.4040200668 & 0 \\
\hline 0.6 & 0.6060301002 & 0.6060301002 & 0 \\
\hline 0.8 & 0.8080401336 & 0.8080401336 & 0 \\
\hline 1.0 & 1.0100501670 & 1.0100501670 & 0 \\
\hline
\end{tabular}




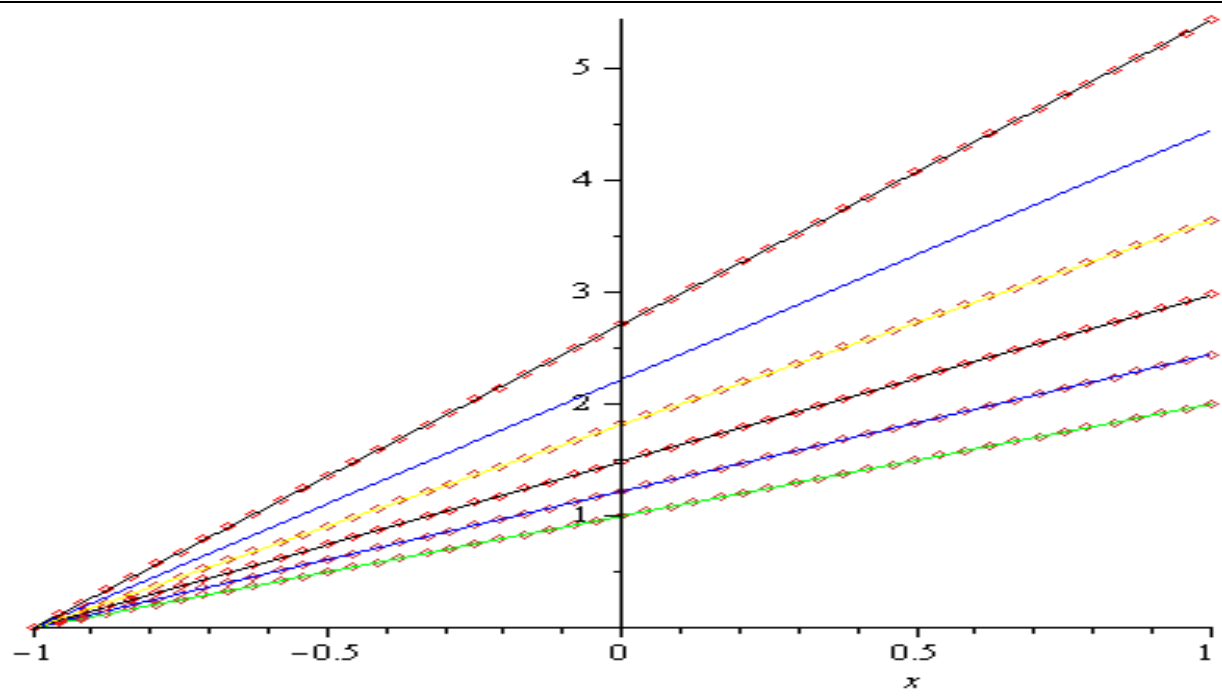

Şekil $5 t=0,0.2,0.4,0.6,0.8,1$ için tam çözüm ve yaklaşı çözüm eğrilerinin karşılaştırması

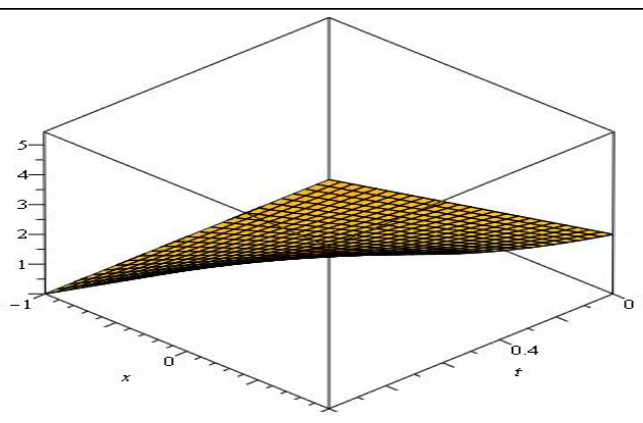

Tam çözüm

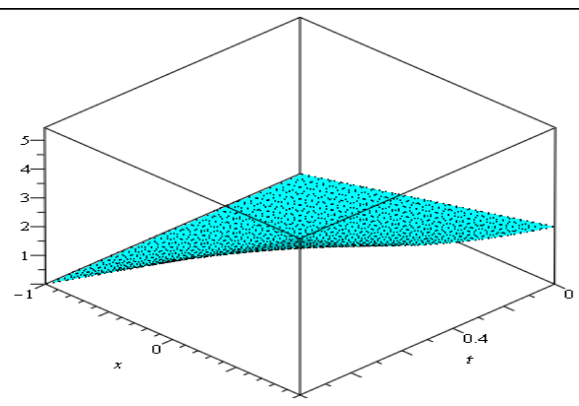

Yaklaşık çözüm

Şekil $6 x$ ve $t$ değerleri için tam çözüm ve yaklaşık çözüm eğrileri

\section{Örnek 4}

$$
\begin{aligned}
& \frac{\partial u}{\partial t}=-\frac{\partial u}{\partial x}(-x)+\frac{\partial^{2} u}{\partial x^{2}}\left(\frac{1-x^{2}}{2}\right), \\
& u(x, 0)=x^{2}+1, \quad x \in R,
\end{aligned}
$$

(11)-(12) Fokker-Planck denkleminde $A(x)=-x, B(x)=\frac{1-x^{2}}{2}$ olan drift ve difüzyon katsayıları sadece $x$ - konum değişkenine bağlıdır ve $f(x)=x^{2}+1$ biçiminde tanımlıdır. Ayrıca (11)-(12) Fokker-Planck denklemi $u(x, t)=\left(x^{2}+1\right) e^{t}$ tam çözümüne sahiptir.

(11)-(12) Fokker-Planck denkleminin indirgenmiş diferansiyel dönüşüm metodu ile çözümü için öncelikle denklemdeki tüm terimlerin aşağıdaki gibi:

$$
\begin{aligned}
& \frac{\partial u}{\partial t} \rightarrow U_{k}(x)=(k+1) U_{k+1}(x), \\
& \frac{\partial u}{\partial x} \rightarrow \frac{\partial U_{k}(x)}{\partial x}, \\
& \frac{\partial^{2} u}{\partial x^{2}} \rightarrow \frac{\partial^{2} U_{k}(x)}{\partial x^{2}}, \\
& x^{2}+1 \rightarrow U_{0}(x)=x^{2}+1,
\end{aligned}
$$


$t$ - değişkenine göre diferansiyel dönüşümleri alınır ve (11) denkleminde yerlerine yazılırsa

$$
U_{k+1}(x)=\frac{1}{k+1}\left[x \frac{\partial U_{k}(x)}{\partial x}+\left(\frac{1-x^{2}}{2}\right) \frac{\partial^{2} U_{k}(x)}{\partial x^{2}}\right],
$$

tekrar bağıntısı elde edilir. $\mathrm{Bu}$ tekrar bağıntısında $k=0,1,2,3, \ldots$ değerleri için aşağıdaki diferansiyel dönüşüm katsayıları 4 iterasyon sonucu bulunur:

$$
U_{0}(x)=x^{2}+1, \quad U_{1}(x)=x^{2}+1, \quad U_{2}(x)=\frac{x^{2}+1}{2 !}, \quad U_{3}(x)=\frac{x^{2}+1}{3 !}, \quad U_{4}(x)=\frac{x^{2}+1}{4 !},
$$

bu diferansiyel dönüşüm katsayıları (1) denkleminde yazılır ve

$$
\begin{aligned}
& u(x, t)=\sum_{k=0}^{\infty} U_{k}(x) t^{k}=x^{2}+1+\left(x^{2}+1\right) t+\left(x^{2}+1\right) \frac{t^{2}}{2 !}+\left(x^{2}+1\right) \frac{t^{3}}{3 !}+\cdots, \\
& u(x, t)=\left(x^{2}+1\right)\left(1+t+\frac{t^{2}}{2 !}+\frac{t^{3}}{3 !}\right)
\end{aligned}
$$

yaklaşı çözümü bulunur.

Yukarıda elde edilen çözümde $t=0.01 \quad 0,0.2,0.4,0.6,0.8,1$ değerleri için tam çözüm değeri ve farklı $x$ 'ler için tam çözüm, ve yaklaşık çözüm eğrileri (aşağıdan yukarı yaklaşık çözüm ve hata değerleri bulunarak doğru) karşılaştırılır. Şekil 8'de ise sırasıyla Tablo 5 ile verilir. Şekil 7 'de $t=$ tam çözüm ve yaklaşık çözüm eğrileri farklı $x$ ve $t$ değerlerine göre çizilir.

Tablo $5 t=0.01$ değeri için tam çözüm, yaklaşık çözüm ve hata değerleri

\begin{tabular}{|c|c|c|c|}
\hline$x$ & Tam çözüm & Yaklaş1k çözüm & Hata \\
\hline-1.0 & 2.020100334 & 2.020100334 & 0 \\
\hline-0.6 & 1.373668227 & 1.373668227 & 0 \\
\hline-0.2 & 1.050452174 & 1.050452174 & 0 \\
\hline 0 & 1.010050167 & 1.010050167 & 0 \\
\hline 0.2 & 1.050452174 & 1.050452174 & 0 \\
\hline 0.6 & 1.373668227 & 1.373668227 & 0 \\
\hline 1.0 & 2.020100334 & 2.020100334 & 0 \\
\hline
\end{tabular}




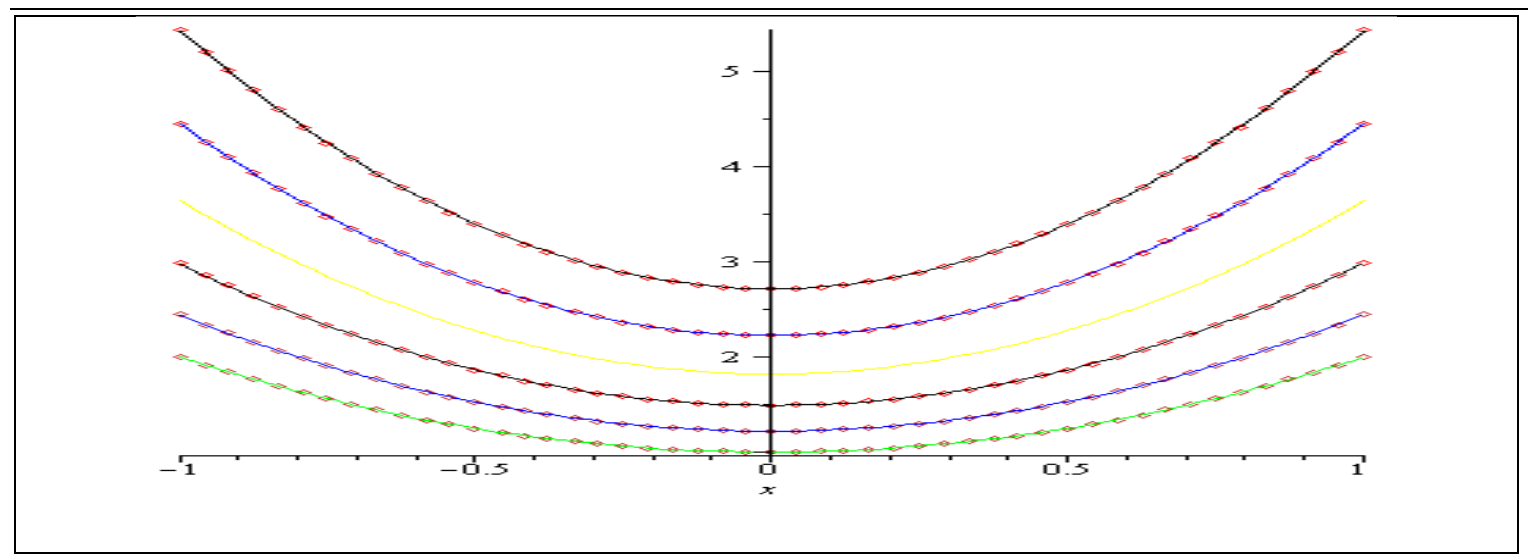

Şekil $7 t=0,0.2,0.4,0.6,0.8,1$ için tam çözüm ve yaklaşık çözüm eğrilerinin karşılaştırması

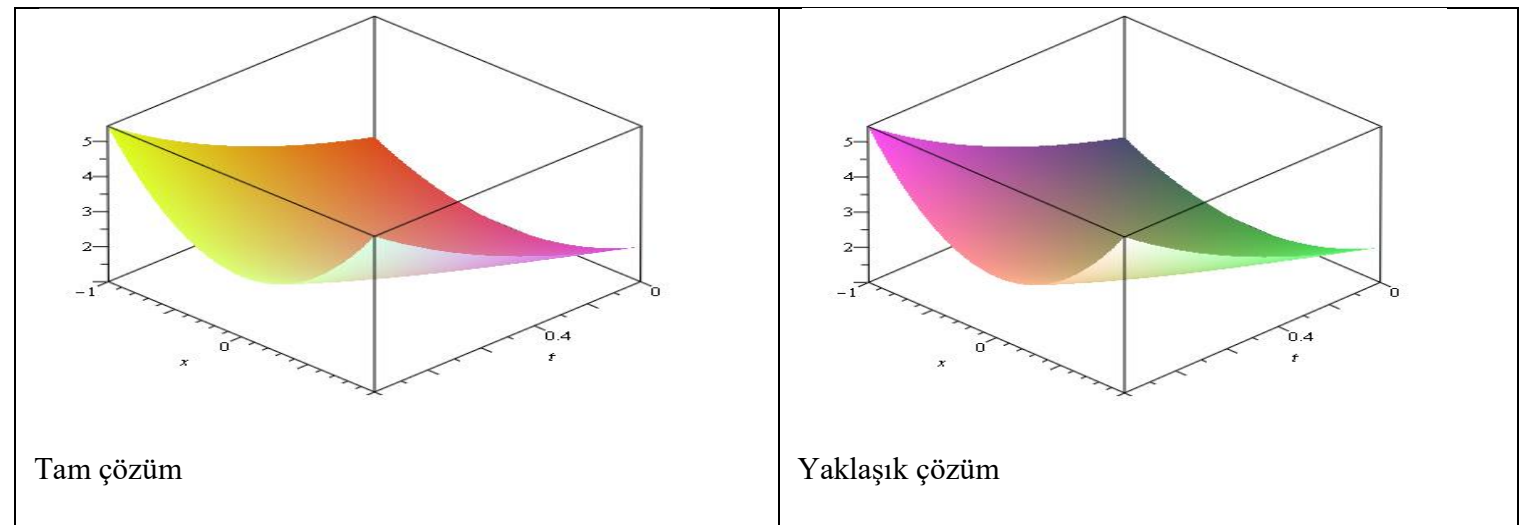

Şekil $8 x$ ve $t$ değerleri için tam ve yaklaşık çözüm eğrileri

\section{Sonuç ve Tartışma}

Fokker-Planck denklemine ilişkin başlangıç değer problemlerinin çözümü için indirgenmiş diferansiyel dönüşüm metodu kullanılarak analitik bir yaklaşım yapılmıştır ve seri çözümler bulunmuştur. $\mathrm{Bu}$ çözümlerden elde edilen sayısal veriler, indirgenmiş diferansiyel dönüşüm metodunun çok iyi performansa sahip olduğunu göstermiştir. Önerilen bu metot, birçok karmaşık lineer ve lineer olmayan adi

\section{Kaynaklar}

Ayaz, F. (2003). "On the Two-dimensional Differential Transform Method", Applied Mathematics and Computation, 1432(2-3), 361-374.

Amiraliyev, G.M., Cimen E., Amirali, I. and Cakir, M. (2017). "High-order Finite Difference Technique for Delay Pseudo-parabolic", Journal of Computational and Applied Mathematics, 321, 1-7.

Amirali, I., Amiraliyev, G.M., Cakir, M. and Cimen, E. (2014). "Explicit Finite Difference Methods for the Delay ve kısmi türevli denklemlerin başlangıç ve sınır değer problemlerine uygulanabilir. En önemlisi de lineerleştirme ve ayrıklaştırma işlemleri gerektirmez. Dolayısıyla sayısal hesaplamaları ciddi anlamda azaltır.

\section{Teşekkür}

Yazar, çalışmanın kalitesinin iyileştirilmesine yönelik olarak değerli hakemlerin yorum, eleştiri ve önerilerine minnettardır.

Pseudoparabolic Equations", The Scientific World Journal, (2014), 7 pages.

Benhammouda, B., Vazquez-Leal, H. and Sarmiento-Reyes, A. (2014). "Modified Reduced Differential Transform Method for Partial DifferentialAlgebraic Equations", Journal of Applied Mathematics, 2014, 9 pages. 
Biazar, J., Gholamin, P. and Hosseini, K. (2010). "Variational Iteration Method for Solving Fokker-Planck Equation", Journal of the Franklin Institute, 347(7), 1137-1147.

Bhrawy, A.H., Ahmed Engy, A. and Baleanu, D. (2014). "An Efficient Collocation Technique for Solving Generalized Fokker-Planck Type Equations with Variable Coefficients", Proceedings of the Romanian Academy, Series A, 15(4), 322-330.

Cakir, M. and Arslan, D. (2015). "The Adomian Decomposition Method and the Differential Transform Method for Numerical Solution of MultiPantograph Delay Differential Equations", Applied Mathematics, 6, 1332-1343.

Cakir, M. and Arslan. D. (2016). "Reduced Differential Transform Method for Sixth-Order Boussinesq Equation", Mathematics and Statistics, 2(2).

Chen, C.K. and Ho, S.H. (1999). "Solving Partial Differential Equations by Two Dimensional Differential Transform Method", Applied Mathematics and Computation, 106, 171-179.

Dreger, J., Pelster A. and Hamprecht, B. (2005). "Variational Perturbation Theory for Fokker-Planck Equation with Nonlinear Drift", European Physical Journal, B45, 355.

Gupta, P.K. (2011). “Approximate Analytical Solutions of Fractional Benney-Lin Equation by Reduced Differential Transform Method and the Homotopy Perturbation Method", Computers \& Mathematics with Applications, 61(9), 2011, 2829-2842.

Haghbin, A. and Hesam, S. (2012). "Reduced Differential Transform Method for Solving Seventh Order Sawada Kotera Equations", The Journal of
Mathematics and Computer Science, 5, 53-59.

Hesam, S., Nazemi A.R. and Haghbin, A. (2012). "Analytical Solution for the Fokker-Planck Equation by Differential Transform Method", Scientia Iranica, B19, 1140.

Hosseinzadeh, H. and Salehpour, E. (2013). "Reduced Differential Transform Method for Solving Partial Differential Equations with Variable Coefficients", Technical Journal of Engineering and Applied Sciences, 3788-3791.

Kazem, S., Rad, J.A. and Parand, K. (2012). "Radial Basis Functions Methods for Solving Fokker-Planck Equation", Engineering Analysis with Boundary Elements, 36, 181-189.

Keskin Y. and Oturanç G. (2010). "Reduced Differential Transform Method for Generalized Kdv Equations", Mathematical and Computational Applications, 15(3), 382-393.

Kım, A.D. and Tranquillı, P. (2008). "Numerical Solution of the FokkerPlanck Equation with Variable Coefficients", Journal of Quantitative Spectroscopy \& Radiative Transfer, 109, 727-740.

Koide, T. (2017). "Perturbative Expansion of Irreversible Work in Fokker-Planck Equation ‘a la Quantum Mechanics”, Journal of Physics A: Mathematical and Theoretical, 50(32).

Kudu, M. and Amiraliyeva, I.G. (2014). "Method of Lines for Third Order Partial Differential Equations", Journal of Applied Mathematics and Physics, 2014(2), 33-36.

Lakestan1, M. and Dehghan, M. (2009). "Numerical Solutions of Fokker-Plank Equation Using the Cubic B-spline Scaling Functions", Numerical Methods Partial Differential Equations, 25, 418-429. 
Mohamed, M.S. and Gepreel, K.A. (2017). "Reduced Differential Transform Method for Nonlinear Integral Member of Kadomtsev-Petviashvili Hierarchy Differential Equations", Journal of the Egyptian Mathematical Society, 25(1), 1-7.

Rawashdeh, M. (2013). "Using the Reduced Differential Transform Method to Solve Nonlinear PDEs Arises in Biology and Physics", World Applied Sciences Journal, 23, 1037-1043.

Narayanan, S. and Kumar, P. (2006). "Solution of Fokker-Planck Equation by Finite Element and Finite Difference Methods for Nonlinear Systems", Sādhanā Academy Proceedings in Engineering sciences, 31(4), 445-461.

Risken, H. (1989). “The Fokker-Plank Equation: Method of Solution and Applications", Springer Verlag, Berlin.

Sadighi, A., Ganji, D.D and Sabzehmeidani, Y. (2007). "A Study on Fokker-Planck Equation by Variational Iteration and Homotopy-perturbation Methods", International Journal of Nonlinear Science, 4 (2007), 92-102.

Saravanan, A. and Magesh, N. (2013). "A Comparison Between the Reduced Differential Transform Method and the Adomian Decomposition Method for the Newell-Whitehead-Segel Equation", Journal of the Egyptian Mathematical Society, 21(3), 259-265.

Srivastava, V.K., Mishra, N., Kumar, S., Singh, B.K. and Awasthi M.K. (2014). "Reduced Differential Transform Method for Solving $(1+n)$ Dimensional Burgers Equation", Egyptian Journal of Basic and Applied Sciences, 1(2), 115-119.

Tatarı, M., Deghan, M. and Razzaghı, M. (2007). "Application of the Adomian Decomposition Method for the Fokker-
Planck Equation", Mathematical and

Computer Modelling, 45, 639-650.

Yıldırım, K., İbiş, B. and Bayram, M. (2012). "New Solutions of the Nonlinear Fisher Type Equations by the Reduced Differential Transform", Nonlinear Science Letters, 3, 29-36.

Zhou, J.K. (1986). "Differential Transformation and its Applications for Electrical Circuits", Huazhong University Press, Wuhan, China. 